\title{
The Observer Error Linearization Problem via Dynamic Compensation
}

\author{
C. Califano, C.H. Moog
}

DOI:10.1109/tac.2014.2308606. In IEEE TRANSACTIONS ON AUTOMATIC CONTROL - ISSN:0018-9286. (c) 2014 IEEE. Personal use of this material is permitted. Permission from IEEE must be obtained for all other users, including reprinting/ republishing this material for advertising or promotional purposes, creating new collective works for resale or redistribution to servers or lists, or reuse of any copyrighted components of this work in other works.

\begin{abstract}
Linearization by output injection has played a key role in the observer design for nonlinear control systems for almost three decades. In this paper, following some recent works, geometric necessary and sufficient conditions are derived for the existence of a dynamic compensator solving the problem under regular output transformation. An algorithm which computes a compensator of minimal order is given.
\end{abstract}

\section{Index Terms}

Linearization by output injection, nonlinear systems, state and output transformations, dynamic compensator.

\section{INTRODUCTION}

In the early 80 's, the transformation of a nonlinear system into a linear canonical observer form was obtained and geometric conditions were derived in [22]. This so-called static solution has been widely investigated both in the continuous and discrete time context (see for example the early works of [6], [23], [21], [33], right to [13], [12], [29], [5], [14], [24], [31], [30], [32] where more general transformations were considered for continuous time systems, and to [26], [11], [37] for continuous systems with delay; [25], [4], [20], [15], [19], [9], [27], [10] deal with discrete-time and sample-data systems). Whenever those conditions are not fulfilled, the problem may be still solvable by using a dynamic compensator. This kind of solution was first considered in the discrete time context in [15] where it was first shown that for autonomous systems, differently from the continuous time case, the observability condition was enough to guarantee the reconstruction of the state through a sufficiently large buffer. The problem was further investigated in [36] and [18] where nonautonomous systems were considered. In continuous time, starting from [17], where a dynamic solution was proposed for the first time by considering the characteristic equation, several dynamic compensators have been proposed in the literature based on different approaches leading to sufficient conditions: in [28] and [35] the problem was addressed by considering chains of integrators connected to the outputs of the system; in [3] a more general setting of the problem was given, while the solution was sought within a special class, consisting of chains of integrators, or linear stable systems; finally in [2] a constructive procedure was proposed for autonomous systems based on the relative degree of the output, while more recently in [7], [8] a geometric approach was used for single output autonomous systems. Despite the numerous works on the topic, the existence of a dynamic solution in continuous time is far from being fully characterized.

In the present paper, given a SISO observable system, necessary and sufficient conditions are given for the existence of a dynamic compensator such that the extended system is equivalent under change of coordinates and regular output transformation to a linear observable system up to input and output injection. Such necessary and sufficient conditions, being algorithm based, are constructive, and lead to the computation of a solution of minimal order

C.Califano is with Dipartimento di Ingegneria Informatica Automatica e Gestionale Antonio Ruberti, Università di Roma La Sapienza, Via Ariosto 25,00185 Italy; califanod dis . uniroma1. it

C.H. Moog is with L'UNAM, IRCCyN, UMR C.N.R.S. 6597, 1 rue de la Noë, BP 92101, 44321 Nantes Cedex 3, France; moog@ieee. org 
whenever it exists. The effective design of an observer, which is beyond the aim of the present paper, is then notably simplified: in fact the state can be estimated through the Kazantzis-Kravaris/Luenberger observer [20] provided the extended system and the coordinates transformation satisfy appropriate assumptions as discussed in detail in [1]. We end this Section by stating exactly the problem under study.

Problem formulation: Given the single output observable system

$$
\begin{aligned}
\dot{x} & =f(x)+g(x) u \\
y & =h(x)
\end{aligned}
$$

with $x \in \mathbb{R}^{n}$ ( $\left.n>1\right), y \in \mathbb{R}, u \in \mathbb{R}, h, f$ and $g$ analytic functions in their argument, find, if possible, an integer $\ell \in \mathbb{N}$, a compensator

$$
\dot{\xi}=\eta_{1}(y, \xi)+\eta_{2}(y, \xi) u, \quad \xi \in \mathbb{R}^{\ell}
$$

and $\ell+1$ independent functions $\tilde{y}_{i}^{e}=\varphi_{i}(y, \xi), i \in[1, \ell+1]$ where $\eta_{1}, \eta_{2}$ and $\varphi_{i}, i \in[1, \ell+1]$ are smooth functions in their arguments, such that the extended system (1), (3), with output function

$$
\tilde{y}^{e}=\left(\tilde{y}_{1}^{e}, \cdots, \tilde{y}_{\ell+1}^{e}\right)^{T}=\left(\varphi_{1}(y, \xi), \cdots, \varphi_{\ell+1}(y, \xi)\right)^{T}=\varphi(y, \xi),
$$

with $\operatorname{rank}\left[\frac{\partial \varphi(y, \xi)}{\partial(y, \xi)}\right]=\ell+1$, is equivalent under change of coordinates $z=\Phi(\xi, x)$ to the observable canonical form

$$
\begin{aligned}
\dot{z} & =A z+\psi_{1}\left(\tilde{y}^{e}\right)+\psi_{2}\left(\tilde{y}^{e}\right) u \\
\tilde{y}^{e} & =\varphi(y, \xi)=C z
\end{aligned}
$$

with $(A, C)$ an observable pair of the form

$$
\begin{aligned}
A & =\operatorname{diag}\left(A_{1} \cdots A_{\ell+1}\right), \quad C=\operatorname{diag}\left(C_{1} \cdots C_{\ell+1}\right), \\
A_{i} & =\left(\begin{array}{ll}
\mathbf{0} & 0 \\
\mathbf{I} & \mathbf{0}
\end{array}\right), \quad C_{i}=\left(\begin{array}{ll}
\mathbf{0} & 1
\end{array}\right)
\end{aligned}
$$

$\psi_{j}\left(\tilde{y}^{e}\right)=\left(\psi_{j 1}\left(\tilde{y}^{e}\right), \cdots, \psi_{j, \ell+1}\left(\tilde{y}^{e}\right)\right)^{T}, j \in 1,2$ and $A_{i}, C_{i}$ of dimension $k_{i} \times k_{i}, 1 \times k_{i}$ respectively for $i \in[1, \ell+1]$. $\triangleleft$

The paper is organized as follows. Standard results for multi-output systems are recalled in Section II since the search for a dynamic post-compensator will transform the single-output problem into a multi-output problem. Section III is devoted to the main results. Practical computations are made through an algorithm in Section III-C and concluding remarks are stated in Section IV.

\section{Recalls AND PReliminary Results}

The following notation will be used. Given a function $\lambda(x)$, its differential is $d \lambda(x)=\left(\frac{\partial \lambda}{\partial x_{1}}, \cdots, \frac{\partial \lambda}{\partial x_{n}}\right)$. Its Lie derivative along a vector field $\tau(x)$, is given by $L_{\tau} \lambda(x):=\frac{\partial \lambda(x)}{\partial x} \tau$. Accordingly $L_{\tau}^{i} \lambda(x)=L_{\tau}\left(L_{\tau}^{i-1} \lambda(x)\right)$. Given the vector fields $\tau_{1}(x), \tau_{2}(x)$, their Lie bracket is defined as $\left[\tau_{1}, \tau_{2}\right]:=a d_{\tau_{1}}\left(\tau_{2}\right)=\frac{\partial \tau_{2}}{\partial x} \tau_{1}-\frac{\partial \tau_{1}}{\partial x} \tau_{2} . \mathcal{C}_{i}^{j}$ will denote the binomial coefficient, that is $\mathcal{C}_{i}^{j}:=\left(\begin{array}{l}j \\ i\end{array}\right)=\frac{j !}{i !(j-i) !}$.

\section{A. Equivalence under change of coordinates for multi-output systems}

The problem formulation recalled in the Introduction underlines that the given single-output system (1), (2) is transformed into the multi-output system (1), (3), (4). Hereafter we thus recall the general result stated in [33] concerning the equivalence of a multi-output system to the linear observer canonical form up to input and output injection, since it will be used to characterize the conditions for the existence of the compensator (3) as well as its computation. Consider then the nonlinear continuous time system

$$
\begin{aligned}
\dot{x} & =f(x)+g(x) u \\
y & =h(x)
\end{aligned}
$$

with $x \in \mathbb{R}^{n}, y \in \mathbb{R}^{p}, u \in \mathbb{R}^{m}, f(x), g_{i}(x)$, for $i \in[1, m]$ and $h_{j}(x)$ for $j \in[1, p]$ analytic functions in their arguments. Without loss of generality, it is assumed that the origin is an equilibrium point for the system, that is $f(0)=0$ and $h(0)=0$. 
Definition 1: The multi-output system (7) is said to be locally observable around $x_{0}=0, u_{0}=0$ with observability indices $k_{i}, i \in[1, p]$, if $\sum_{i=1}^{p} k_{i}=n$ and the observability matrix

$$
O=\left(\left(d h_{1}\right)^{T} \ldots\left(d L_{f}^{k_{1}-1} h_{1}\right)^{T} \ldots\left(d h_{p}\right)^{T} \ldots\left(d L_{f}^{k_{p}-1} h_{p}\right)^{T}\right)^{T}
$$

has full rank $n$ locally around $x_{0}=0$.

The problem of defining a diffeomorphism $z=\Phi(x)$ which transforms (7) into the linear observable canonical form up to input and output injection ([16]),

$$
\begin{aligned}
\dot{z} & =A z+\psi_{1}(y)+\psi_{2}(y) u \\
y & =C z
\end{aligned}
$$

where $A$ and $C$ are of the form (6) with $\ell+1=p$ (and, for $i \in[1, p], A_{i}$ and $C_{i}$ of dimension $k_{i} \times k_{i}$ and $k_{i} \times 1$ respectively), is linked to the existence of $p$ vector fields $r_{i 1}(x), i \in[1, p]$ solutions of

$$
d\left(L_{f}^{\mu} h_{j}\right) r_{i 1}=0, \mu \in\left[0, k_{i}-2\right], d\left(L_{f}^{k_{i}-1} h_{j}\right) r_{i 1}=\delta_{i j}
$$

$\forall j \in[1, p]$. In (9), $\delta_{i j}$ denotes the Kronecker index which is equal to 1 if $i=j$ and 0 otherwise. The following theorem is shown in [33], using the notations $\mathcal{O}=\left\{d h_{i} \cdots d L_{f}^{k_{i}-1} h_{i}, i \in[1, p]\right\}$, $\mathcal{O}_{i}=\left\{d h_{i}, \cdots, d L_{f}^{k_{i}-2} h_{i}, d h_{j} \cdots d L_{f}^{k_{i}-1} h_{j}, j \neq i, j \in[1, p]\right\}^{1}$ and $r_{j k}=-a d_{f}\left(r_{j, k-1}\right)=\left[r_{j, k-1}, f\right]$.

Theorem 1: [33] There exists a diffeomorphism $z=\Phi(x)$ which transforms system (7) into the linear observable canonical form up to input and output injection (8) if and only if

i) The system is observable $\left(\operatorname{rank} O=n\right.$ ) with observability indices $k_{i}, i \in[1, p]$

ii) $\operatorname{span} \mathcal{O}_{i} \equiv \operatorname{span}\left(\mathcal{O}_{i} \cap \mathcal{O}\right)$

iii) $\left[r_{j k}, r_{i \eta}\right]=0 \quad \forall j, i \in[1, p], k \in\left[1, k_{j}\right], \eta \in\left[1, k_{i}\right]$

iv) $\left[g_{j}, r_{i \eta}\right]=0 \quad \forall j \in[1, m], \forall i \in[1, p], \eta \in\left[1, k_{i}-1\right]$

\section{B. A preliminary technical result}

Next result will be instrumental in the characterization and computation of a solution if it exists.

Proposition 1: Assume that for the given observable dynamics (1), (2), there exists a dynamic compensator of dimension $\ell$ and of the form (3), such that the extended system (1), (3) can be rewritten in appropriate coordinates $z=$ $\Phi(x, \xi)$ in the form (5), with respect to the $\ell+1$ fictitious independent functions $\tilde{y}^{e}=\left(\varphi_{1}(y, \xi), \cdots, \varphi_{\ell+1}(y, \xi)\right)^{T}$, with $\operatorname{rank}\left(\frac{\partial \varphi(y, \xi)}{\partial(y, \xi)}\right)=\ell+1$. Let, without loss of generality, the first output satisfy $\frac{\partial \varphi_{1}(y, \xi)}{\partial y} \neq 0$. Then the extended dynamics (1), (3) admits the observer canonical form up to input and output injection also with respect to the extended output $\hat{y}^{e}=\left(\varphi_{1}(y, \xi), \xi_{1}, \cdots, \xi_{\ell}\right)^{T}$ with observability indices $(n, 1, \cdots, 1)$.

Proof: Since the system admits the observer canonical form up to input and output injection then it satisfies Theorem 1. Since $\operatorname{span}\left\{d \tilde{y}^{e}\right\} \equiv \operatorname{span}\{d y, d \xi\}$ and the observability index $k_{1}=n$ (since $\varphi_{1}(y, \xi)$ depends on $y$ ), one immediately verifies that ii) of Theorem 1 is satisfied only if $\varphi_{j}(y, \xi)=\varphi_{j}(\xi)$ for $j \in[2, \ell+1]$, since otherwise $\operatorname{span}\left(\mathcal{O}_{1}\right) \not \equiv \operatorname{span}\left(\mathcal{O}_{1} \cap \mathcal{O}\right)$. As a consequence, the output function $\tilde{y}^{e}=\left(\varphi_{1}(y, \xi), \hat{\varphi}(\xi)^{T}\right)$, where $\hat{\varphi}(\xi): \mathbb{R}^{\ell} \rightarrow \mathbb{R}^{\ell}$ is invertible and has observability indices $\left(k_{1}, k_{2}, \cdots, k_{\ell+1}\right)=(n, 1, \cdots, 1)$. Let $\left(\chi^{T}, z_{1}^{T}\right)^{T}=$ $\left(\hat{\phi}_{0}(x, \xi)^{T}, \phi_{1}(x, \xi)^{T}\right)^{T}$, be the associated change of coordinates with $\operatorname{rank} \frac{\partial\left(\phi_{1}(x, \xi)\right)}{\partial x}=n$ and $\tilde{y}_{1}^{e}=C z_{1}$. The proof ends by taking $\hat{y}^{e}=\left(\varphi_{1}(y, \xi), \xi_{1}, \cdots, \xi_{\ell}\right)^{T}$, and by considering the coordinates $z=\left(\tilde{\xi}^{T}, z_{1}^{T}\right)^{T}$, in which the system reads (5).

\section{MAIN RESUltS}

Since according to Proposition 1, if a solution exists then we can take as output functions $\tilde{y}_{1}^{e}=\varphi(y, \xi), \tilde{y}_{j+1}^{e}=\xi_{j}$ $j \in[1, \ell]$, we will first investigate the relationship between the $r_{i}(x)$ 's $i \in[1, n]$ defined on the original system with respect to the single output $y=h(x)$ and the $\tilde{r}_{i j}^{e}(x, \xi)$ 's $i \in[1, \ell+1], j \in\left[1, k_{i}\right]$ defined on the extended system with respect to the transformed output functions $\tilde{y}^{e}$ defined above. This will yield the characterization of the output transformation and the dynamic compensator. The following holds true:

\footnotetext{
${ }^{1}$ Note that for $k_{i}=1, \mathcal{O}_{i}$ reduces to $\mathcal{O}_{i}=\left\{d h_{j}, j \neq i, j \in[1, p]\right\}$
} 
Proposition 2: Given system (1-2), let $r_{1}(x)$ be the vector field solution of equation (9) with respect to the given output function $y=h(x)$. Consider the extended system (1), (3), with drift $F^{e}(x, \xi)=\left(\eta_{1}^{T}(y, \xi) \quad f^{T}(x)\right)^{T}$ and output functions $\tilde{y}_{1}^{e}=\tilde{h}_{1}^{e}=\varphi(y, \xi), \tilde{y}_{i+1}^{e}=\tilde{h}_{i+1}^{e}=\xi_{i}, i \in[1, \ell]$, where $\frac{\partial \varphi(y, \xi)}{\partial y} \neq 0$ with observability indices $\tilde{k}_{1}=n, \tilde{k}_{i+1}=1$ for $i \in[1, \ell]$. Then the vector fields $\tilde{r}_{i 1}^{e}(x, \xi), i \in[1, \ell+1]$, solutions of the corresponding equations (9) $\forall j \in[1, \ell+1]$ exist and are given by

$$
\begin{aligned}
& \tilde{r}_{11}^{e}(x, \xi)=\left[\frac{\partial \varphi(y, \xi)}{\partial y}\right]^{-1} r_{1}^{T} \frac{\partial}{\partial x}=\alpha(y, \xi) r_{1}^{T} \frac{\partial}{\partial x} \\
& \tilde{r}_{\mu, 1}^{e}=\frac{\partial}{\partial \xi_{\mu-1}}+\sum_{i=1}^{n} \gamma_{\mu-1, i}(x, \xi) r_{i}(x)^{T} \frac{\partial}{\partial x} \quad \mu \in[2, \ell+1]
\end{aligned}
$$

with $\gamma_{\mu-1, n}=-\left[\frac{\partial \varphi(y, \xi)}{\partial y}\right]^{-1} \frac{\partial \varphi(y, \xi)}{\partial \xi_{\mu-1}}$. Accordingly, for $j \in[2, n]$,

$$
\tilde{r}_{1 j}^{e}(x, \xi)=\sum_{i=0}^{j-1} \mathcal{C}_{i}^{j-1}(-1)^{j-1-i} L_{F^{e}(x, \xi)}^{j-i-1} \alpha(x, \xi) r_{i+1}^{T}(x) \frac{\partial}{\partial x}
$$

Proof: Since the original single output system is observable, $r_{1}(x)$ exists and is unique; moreover $r_{1}(x), \cdots, r_{n}(x)$ are independent ([23]). They can thus be used as a basis in $\mathbb{R}^{n}$. Consider now the extended system (1), (3) with extended output $\tilde{y}^{e}=\left(\varphi(y, \xi), \xi_{1}, \cdots, \xi_{\ell}\right)^{T}$, which has, by Proposition 1 , observability indices $(n, 1, \cdots, 1)$. The corresponding vector fields $\tilde{r}_{\mu 1}^{e}, \mu \in[1, \ell+1]$, solution to (9), if they exist can be expressed as $\tilde{r}_{\mu 1}^{e}=$ $\sum_{i=1}^{n} \alpha_{\mu i}(x, \xi) r_{i}(x)^{T} \frac{\partial}{\partial x}+\sum_{j=1}^{\ell} \beta_{\mu j}(x, \xi) \frac{\partial}{\partial \xi_{j}}$, in the basis $\left(r_{1}^{T}(x) \frac{\partial}{\partial x}, \cdots, r_{n}^{T}(x) \frac{\partial}{\partial x}, \frac{\partial}{\partial \xi_{1}}, \cdots, \frac{\partial}{\partial \xi_{\ell}}\right)$.

Since $\tilde{k}_{1}^{e}=n, d\left(L_{F^{e}}^{\nu} \tilde{y}^{e}\right) \tilde{r}_{11}^{e}(x, \xi)=0$ for $0 \leq \nu \leq n-2$, which implies immediately that $\beta_{1 j}(x, \xi)=0$ for $j \in[1, \ell]$ and that $\alpha_{1 i}(x, \xi)=0$ for $i \in[2, n]$. Finally, since for $j \in[2, \ell+1], \nu \in[1, n-1], d\left(L_{F e}^{\nu} \tilde{y}_{j}^{e}\right) \in$ $\operatorname{span}\left\{d \xi, d \tilde{y}_{1}^{e}, \cdots, d\left(L_{F^{e}}^{\nu-1} \tilde{y}_{1}^{e}\right)\right\}$ the previous relation ensures also that $d\left(L_{F^{e}}^{\nu} \tilde{y}_{j}^{e}\right) \tilde{r}_{11}^{e}(x, \xi)=0, j \in[2, \ell+1], \nu \in$ $[1, n-1]$ while $d\left(L_{F e}^{n-1} \tilde{y}_{1}^{e}\right) \tilde{r}_{11}^{e}=1$ implies that $\frac{\partial \varphi(y, \xi)}{\partial y} d\left(L_{f}^{n-1} h(x)\right) r_{1}(x) \alpha_{11}(x, \xi)=\frac{\partial \varphi(y, \xi)}{\partial y} \alpha_{11}(x, \xi)=1$. As a consequence $\alpha_{11}(x, \xi)=\left[\frac{\partial \varphi(y, \xi)}{\partial y}\right]^{-1}$ that is $\tilde{r}_{11}^{e}$ exists and is given by $(10)$, where $\alpha(x, \xi)=\alpha_{11}(x, \xi)=$ $\left[\frac{\partial \varphi(y, \xi)}{\partial y}\right]^{-1}$

In a similar way, for $\mu \in[2, \ell+1]$ since $\tilde{k}_{\mu}^{e}=1$, then it is easily verified that $\tilde{r}_{\mu 1}^{e}(x, \xi)$ exists and must satisfy $d \tilde{y}_{j}^{e} \tilde{r}_{\mu 1}^{e}(x, \xi)=\delta_{\mu j}$. Then necessarily $\beta_{\mu j}(x, \xi)=0$ for $\mu \neq j+1$ and $\beta_{j+1, j}=1$. Moreover since $d \tilde{y}_{1}^{e} \tilde{r}_{\mu 1}^{e}=0$ then $\frac{\partial \varphi(y, \xi)}{\partial y} d h(x) r_{n}(x) \alpha_{\mu n}(x, \xi)+\frac{\partial \varphi(y, \xi)}{\partial \xi_{\mu-1}}=0$ so that $\alpha_{\mu n}(x, \xi)=-\left[\frac{\partial \varphi(y, \xi)}{\partial y}\right]^{-1} \frac{\partial \varphi(y, \xi)}{\partial \xi_{\mu-1}}$ which proves (11). Finally (12) is proven iteratively by using the fact that $\tilde{r}_{1 j}^{e}(x, \xi)=-a d_{F^{e}(x, \xi)} \tilde{r}_{1, j-1}^{e}(x, \xi)$.

Proposition 2 generalizes and includes the particular cases treated in [3], [8].

Next result characterizes the link between the given system and the dynamic compensator solving the problem.

Theorem 2: Assume that the observable system (1), (2), can be transformed into the canonical observer form up to input and output injection through the compensator (3) with respect to the extended output $\tilde{y}^{e}=\left(\varphi(y, \xi), \xi_{1}, \cdots, \xi_{\ell}\right)^{T}$, where $\frac{\partial \varphi(y, \xi)}{\partial y} \neq 0$. Let $F^{e}=\left(\eta_{1}^{T}, f^{T}\right)^{T}$ and $G^{e}=\left(\eta_{2}^{T}, g^{T}\right)^{T}$ be the vector fields characterizing the extended system (1), (3), and consider the output vector $H(x, \xi)=\left(h(x), \xi_{1}, \cdots, \xi_{\ell}\right)^{T}$. Denote by

$$
\beta(y, \xi)=\left(\beta_{y}, \beta_{\xi_{1}}, \cdots, \beta_{\xi_{\ell}}\right)=d\left(\log \left(\frac{\partial \varphi(y, \xi)}{\partial y}\right)\right),
$$

and by $\beta^{(\mu)}=\left(L_{F^{e}}^{\mu} \beta^{T}\right)^{T}$. Then the following properties hold true

$$
\text { i.) } \begin{aligned}
{\left[r_{1}(x), r_{j}(x)\right] } & =0 \quad \forall j \leq n-1 \\
{\left[r_{j}(x), r_{n}(x)\right] } & =\sum_{i=0}^{j-1} \Gamma_{j-i}^{j}(x) r_{j-i}(x), \forall j \in[1, n-1]
\end{aligned}
$$


where, for $j>i$,

$$
\begin{gathered}
\Gamma_{j-i}^{j}=(-1)^{i} \sum_{k=0}^{i} \mathcal{C}_{j-1-i}^{j-1-k} \beta^{(i-k)} Q_{j}^{k}\left(F^{e}, H\right) \\
Q_{j}^{k}\left(F^{e} H\right)=(-1)^{n-j} \mathcal{C}_{n-j+k}^{n-1} d\left(L_{F^{e}}^{n-j+k} H\right) r_{j}^{T} \frac{\partial}{\partial x}-\mathcal{C}_{k}^{j-1} d\left(L_{F e}^{k} H\right) r_{n}^{T} \frac{\partial}{\partial x} \\
\text { ii.) }\left[r_{j}(x), g(x)\right]=\sum_{i=0}^{j-1} \mathcal{C}_{i}^{j-1} \Theta_{i}(x) r_{j-i}(x), \forall j \in[1, n-1] \\
\text { where } \Theta_{i}=(-1)^{i+1} L_{G^{e}} L_{F e}^{i}\left(\log \left(\frac{\partial \varphi(y, \xi)}{\partial y}\right)\right)
\end{gathered}
$$

Proof: By assumption, the extended system (1), (3), with output functions $\tilde{y}_{1}^{e}=\varphi(y, \xi)$, and $\tilde{y}_{i+1}^{e}=\xi_{i}$ for $i \in$ $[1, \ell]$, is equivalent under change of coordinates to the observable canonical form up to input and output injection. As a consequence the vector fields $\tilde{r}_{1 j}^{e}(x, \xi), j \in[1, n]$ given by (10) and (12) must satisfy the condition $\left[\tilde{r}_{1 j}^{e}, \tilde{r}_{1 \mu}^{e}\right]=0$, $\forall j, \mu \in[1, n]$. According to Proposition 2, by construction denoting by $v(y, \xi)=[\alpha(y, \xi)]^{-1}=\frac{\partial \varphi(y, \xi)}{\partial y}$

$$
\begin{aligned}
& r_{1}(x)^{T} \frac{\partial}{\partial x}=\left[\frac{\partial \varphi(y, \xi)}{\partial y}\right] \tilde{r}_{11}^{e}=v(y, \xi) \tilde{r}_{11}^{e}(y, \xi) \\
& r_{j}(x)^{T} \frac{\partial}{\partial x}=\sum_{i=0}^{j-1} \mathcal{C}_{i}^{j-1}(-1)^{j-1-i} L_{F e}^{j-i-1} v(y, \xi) \tilde{r}_{1, i+1}^{e}(y, \xi)
\end{aligned}
$$

As a consequence, one gets that setting $H=\left(h(x), \xi_{1}, \cdots, \xi_{\ell}\right)^{T}$, considering $\beta(y, \xi)$ given by (13) and denoting by $\beta^{(j)}=\left(L_{F^{e}}^{j} \beta^{T}\right)^{T}$, standard computations lead to

$$
\begin{gathered}
{\left[r_{j}^{T} \frac{\partial}{\partial x}, r_{\mu}^{T} \frac{\partial}{\partial x}\right]=} \\
\sum_{p=0}^{\mu-1} r_{\mu-p}^{T} \frac{\partial}{\partial x}(-1)^{p} \mathcal{C}_{\mu-1-p}^{\mu-1} \sum_{k=0}^{p} \mathcal{C}_{k}^{p} \beta^{(p-k)} d\left(L_{F^{e}}^{k} H\right) r_{j}^{T} \frac{\partial}{\partial x} \\
-\sum_{i=0}^{j-1} r_{j-i}^{T} \frac{\partial}{\partial x}(-1)^{i} \mathcal{C}_{j-1-i}^{j-1} \sum_{k=0}^{i} \mathcal{C}_{k}^{i} \beta^{(i-k)} d\left(L_{F e}^{k} H\right) r_{\mu}^{T} \frac{\partial}{\partial x}
\end{gathered}
$$

Since by construction $d\left(L_{F}^{k} H\right) r_{\mu}^{T} \frac{\partial}{\partial x}=0$ for $\mu+k<n$ we immediately get that $\left[r_{1}, r_{j}\right]=0 \forall j \leq n-1$ that is (14), while specifying (22) for $\mu=n$ and considering $Q_{j}^{k}\left(F^{e}, H\right)$ given by (17), one gets $\forall j<n$,

$$
\left[r_{j}, r_{n}\right]=\sum_{i=0}^{j-1}(-1)^{i} r_{j-i} \sum_{k=0}^{i} \mathcal{C}_{j-1-i}^{j-1-k} \beta^{(i-k)} Q_{j}^{k}\left(F^{e}, H\right)
$$

that is (15), with $\Gamma_{j-i}^{j}(x)$ given by (16).

As for ii.), by assumption for $j \in[1, n-1],\left[\tilde{r}_{1 j}^{e}, G^{e}\right]=0$, so that one immediately gets that for $j \in[1, n-1]$,

$$
\sum_{i=0}^{j-1} \mathcal{C}_{i}^{j-1}(-1)^{j-1-i}\left(L_{F e}^{j-i-1} \alpha(\xi, y)\left[r_{i+1}, g\right]-L_{G^{e}} L_{F e}^{j-i-1} \alpha(\xi, y) r_{i+1}\right)=0
$$

that is $\left(\left[r_{1}, g\right], \cdots,\left[r_{n-1}, g\right]\right) \Omega=\left(r_{1}, \cdots, r_{n-1}\right) \Pi$

with $\Omega$ and $\Pi$ two upper triangular matrices of dimension $n-1$ whose generic coefficient in position $(i, j)$ is respectively $\Omega_{i j}=(-1)^{i+j} \mathcal{C}_{j-i}^{j-1} L_{F e}^{j-i} \alpha(y, \xi)$ and $\Pi_{i j}=(-1)^{i+j} \mathcal{C}_{j-i}^{j-1} L_{G^{e}} L_{F^{e}}^{j-i} \alpha(y, \xi)$. $\Omega$ being invertible since $\operatorname{det}(\Omega)=\alpha(y, \xi)^{n-1} \neq 0$, one gets that

$$
\left(\left[r_{1}, g\right], \cdots,\left[r_{n-1}, g\right]\right)=\left(r_{1}, \cdots, r_{n-1}\right) \Pi \Omega^{-1}=T
$$

with $T$ again an upper triangular matrix with generic coefficient in position $(i, j)$ given by $t_{i j}=(-1)^{i+j} \mathcal{C}_{j-i}^{j-1} L_{G^{e}} L_{F e}^{j-i} L o g(\alpha)$; ii.) follows being

$$
\left[r_{i}, g\right]=\sum_{j=0}^{i-1}(-1)^{j+1} \mathcal{C}_{j}^{i-1} L_{G^{e}} L_{F e}^{j}\left(\log \left(\frac{\partial \varphi(y, \xi)}{\partial y}\right)\right) r_{i-j} .
$$


Equations (15)-(16) and (18)-(19) are at the basis of the computation of the output transformation and the dynamic compensator solving the problem as highlighted in the following paragraphs.

\section{A. The output transformation properties}

Proposition 3: Assume that there exists a compensator of dimension $\ell$ of the form (3), and an output transformation $\varphi(y, \xi)$, with $\frac{\partial \varphi(y, \xi)}{\partial y} \neq 0$, such that the extended system (1), (3) can be put in the canonical observer form (5), with respect to the extended output $\tilde{y}^{e}=\left(\varphi(y, \xi), \xi_{1}, \cdots, \xi_{\ell}\right)^{T}$. Let $\beta(y, \xi)=\left[\beta_{y}, \beta_{\xi_{1}}, \cdots, \beta_{\xi_{\ell}}\right]$ be the vector satisfying (15). Then there exists a $\beta_{0}(\xi)$ such that $\beta(y, \xi)$ satisfies

$$
\beta_{y}(y, \xi)=\beta_{y}(y), \text { and } \beta_{\xi_{i}}(y, \xi)=\beta_{\xi_{i}}(\xi)=\frac{\partial \beta_{0}(\xi)}{\partial \xi_{i}} i \in[1, \ell],
$$

and setting $\beta_{I, y}=\int \beta_{y}(y) d y$, the output transformation $\varphi(y, \xi)$ satisfies the relation

$$
\frac{\partial \varphi(y, \xi)}{\partial y}=e^{\beta_{I, y}(y)+\beta_{0}(\xi)} c_{0}
$$

Proof: Setting $H=\left(h(x), \xi_{1}, \cdots, \xi_{\ell}\right)^{T}, d\left(L_{F e}^{n-j} H\right) r_{j}^{T} \frac{\partial}{\partial x}=\left(\begin{array}{llll}1 & 0 & \cdots & 0\end{array}\right)^{T}$, so that in (15), the coefficient of $r_{j}, \Gamma_{j}^{j}$, depends only on $x$ and is given by

$$
\Gamma_{j}^{j}(x)=\beta_{y}(y, \xi)\left[(-1)^{n-j} \mathcal{C}_{n-j}^{n-1}-1\right]
$$

As a consequence $\beta_{y}(y, \xi)=\beta_{y}(y)=\frac{\partial}{\partial y}\left(\log \left(\frac{\partial \varphi(y, \xi)}{\partial y}\right)\right)$. Denoting by $\beta_{I, y}(y)=\int \beta_{y}(y) d y$, we thus have that $\log \left(\frac{\partial \varphi(y, \xi)}{\partial y}\right)=\beta_{I, y}(y)+\beta_{0}(\xi)+c$ which implies (25).

\section{B. The dynamic compensator properties}

In this section, it is shown that whenever the problem is solvable, the post-compensator can always be chosen to be (almost) linear.

Proposition 4: Assume that there exists a compensator of dimension $\ell$ of the form (3) and an output transformation $\varphi(y, \xi)$, with $\frac{\partial \varphi(y, \xi)}{\partial y} \neq 0, y=h(x) \in \mathbb{R}$, such that the extended system (1), (3) can be put in the canonical observer form, with respect to the extended output $\tilde{y}^{e}=\left(\varphi(y, \xi), \xi_{1}, \cdots, \xi_{\ell}\right)^{T}$. Then there exists also a dynamic compensator of dimension $\bar{\ell} \leq \ell$, with $\bar{\ell} \leq n-1$, and of the form

$$
\begin{aligned}
\dot{\chi}_{i} & =\chi_{i+1}+\tilde{\eta}_{1 i}(y)+\tilde{\eta}_{2 i}(y) u, \quad i=1, \cdots, \bar{\ell}-1 \\
\dot{\chi}_{\bar{\ell}} & =\eta_{0, \bar{\ell}}(\chi)+\tilde{\eta}_{1 \bar{\ell}}(y)+\tilde{\eta}_{2, \bar{\ell}}(y) u
\end{aligned}
$$

which solves the problem with respect to the extended output $\bar{y}^{e}=\left(\bar{\varphi}(y, \chi), \chi_{1}, \cdots, \chi_{\bar{\ell}}\right)^{T}$, where $\frac{\partial \bar{\varphi}}{\partial y}=\left.\frac{\partial \varphi(y, \xi)}{\partial y}\right|_{\beta_{0}(\xi)=\chi_{1}}=$ $e^{\beta_{I, y}(y)} e^{\chi_{1}} c_{0}$.

The proof, detailed in the Appendix, is based on the computation of an appropriate change of coordinates such that the dynamic compensator solving the problem is split into a subsystem necessary to solve the problem, and an additional part which can be neglected. In these coordinates the first subsystem has the structure (27).

Remark. The proof of Proposition 4 shows also that the presence of the control in the dynamics may influence the dimension of the compensator. For instance it is sufficient to consider second order systems: in the autonomous case (see [8], [34]) if the problem can be solved then a static solution surely exists whereas this is not true anymore in the nonautonomous case. As an example consider the system $\dot{x}_{1}=x_{2}+x_{1} x_{2} u, \dot{x}_{2}=x_{1}+x_{2} u, y=x_{2}$, which does not admit any static solution but can be put in the canonical observer form by considering the compensator $\dot{\chi}=-y u$ and taking as extended output $\tilde{y}=\left(e^{\chi} y, \chi\right)^{T}$, computed by applying the algorithm in Section III-C. $\triangleleft$ 


\section{The computation of the solution}

Based on Theorem 2 and Propositions 3 and 4, an algorithm is now given for the computation of the desired compensator, if it exists. ${ }^{2}$

Step 0. Solve equations (9) and check if $\left[r_{1}, r_{j}\right]=0$ for $j \leq n-1$. If not go to step exit, else set for $0 \leq l \leq p \leq i<j$

$$
\mathcal{L}_{i}^{j}(p, l)=(-1)^{n-j} \mathcal{C}_{n-j+i}^{n-1} \mathcal{C}_{n-j+l}^{n-j+p} d\left(L_{f}^{n-j+l} h\right) r_{j}-\mathcal{C}_{i}^{j-1} \mathcal{C}_{l}^{p} d\left(L_{f}^{l} h\right) r_{n}
$$

and go to Step 1.

Step 1. Let $j$ be the smallest index such that $\left[r_{j}, r_{n}\right] \neq 0$ and $\mathcal{L}_{0}^{j}(0,0) \neq 0$. Verify that $\Gamma_{j}^{j} / \mathcal{L}_{0}^{j}(0,0)=\beta_{y}(y)$. If it isn't a function of $y$ only, then go to step exit, else compute $\beta_{I, y}(y)=\int \beta_{y}(y) d y$; set $\alpha(y)=e^{-\beta_{I, y}(y)}$, and consider $\tilde{r}_{1}(x)=\alpha(y) r_{1}(x)$, which corresponds to consider the new output $\tilde{y}=\varphi(y)=\int e^{\beta_{I, y}(y)} d y$. Compute the corresponding $\tilde{r}_{i} i \in[2, n]$, accordingly the corresponding $\tilde{\mathcal{L}}_{i}^{j}(p, l)$, for $0 \leq l \leq p \leq i<j$, and go to Step 2 .

Step 2. Check if for all $j \in[1, n-1],\left[\tilde{r}_{j}, \tilde{r}_{n}\right]=0$ and $\left[\tilde{r}_{j}, g\right]=0$. If the conditions are satisfied then the system can be transformed into the canonical observer for up to input output injection with a compensator of order 0 , else if for some $j \in[1, n-1], \tilde{\Gamma}_{j}^{j} \neq 0$ then go to step exit else set $\alpha\left(\tilde{y}, \chi_{1}\right)=e^{-\chi_{1}}, k=0$, and go to Step 3 .

Step 3. Set $i=k+1$; update the compensator adding the dynamics

$$
\dot{\chi}_{i}=\eta_{0 i}(\chi)+\eta_{1 i}(\tilde{y})+\eta_{2 i}(\tilde{y}) u
$$

Accordingly set $F^{e}=\left(\chi_{2}+\eta_{11}(\tilde{y}), \cdots, \eta_{0 i}(\chi)+\eta_{1 i}(\tilde{y}), f^{T}(x)\right)^{T}, G^{e}=\left(\eta_{21}(\tilde{y}), \cdots, \eta_{2 i}(\tilde{y}), g^{T}(x)\right)^{T}$

Step 3a For the computation of $\eta_{1 i}(\tilde{y})$ : for some $j>i$ such that $\tilde{\mathcal{L}}_{i}^{j}(0,0) \neq 0$, verify that

$$
\left((-1)^{i} \tilde{\Gamma}_{j-i}^{j}(\cdot)-\sum_{p=1}^{i-1 \geq 1} \sum_{l=0}^{p} L_{f}^{p-l}\left(\frac{\partial \eta_{1, i-p}(\tilde{y})}{\partial \tilde{y}}\right) \tilde{\mathcal{L}}_{i}^{j}(p, l)\right) / \tilde{\mathcal{L}}_{i}^{j}(0,0)=\mathcal{P}_{i}(\tilde{y})
$$

if it isn't a function of $\tilde{y}$ only, go to Step exit, else set $\eta_{1, i}(\tilde{y})=\int \mathcal{P}_{i}(\tilde{y}) d \tilde{y}$. If with such a choice, considering $Q_{j}^{i}\left(F^{e}, \chi_{1}\right)$, defined by (17), for all $j \in[i+1, n-1], \tilde{\Gamma}_{j-i}^{j}(\cdot)=(-1)^{i} Q_{j}^{i}\left(F^{e}, \chi_{1}\right)$ go to Step $3 b$, else go to Step exit.

Step 3b For the computation of $\eta_{2 i}(\tilde{y})$ : compute $\left[\tilde{r}_{i}, g\right]$; check if

$$
\left[\tilde{r}_{i}, g\right]=\sum_{j=0}^{i-1} \mathcal{C}_{j}^{i-1} \tilde{r}_{i-j} \tilde{\Theta}_{j}, \tilde{\Theta}_{j}=(-1)^{j+1} L_{G^{e}} L_{F^{e}}^{j} \chi_{1}, j \in[0, i-1]
$$

and compute $\eta_{2 i}=(-1)^{i} \tilde{\Theta}_{i-1}-\sum_{j=1}^{i-1 \geq 1} L_{g(x)} L_{f(x)}^{i-1-j} \eta_{1 j}(\tilde{y})$.

If $\eta_{2 i}:=\eta_{2 i}(\tilde{y})$ then go to Step 4, else go to Step exit.

Step 4. Verify if there exists $\eta_{0, i}(\chi)$ such that with the new compensator $\forall j \in[i+1, n-1], l \in[0, j-1]$, $\tilde{\Gamma}_{j-l}^{j}=(-1)^{l} Q_{j}^{l}\left(F^{e}, \chi_{1}\right)$ with $Q_{j}^{l}\left(F^{e}, \chi_{1}\right)$ defined by $(17)$, and

$$
\left[\tilde{r}_{j}, g\right]=\sum_{l=0}^{j-1} \mathcal{C}_{l}^{j-1} \tilde{r}_{j-l} \Theta_{l}, \text { with } \Theta_{l}=(-1)^{l+1} L_{G^{e}} L_{F^{e}}^{l} \chi_{1}
$$

If yes go to Step 5, else if $i \leq n-2$, set $\eta_{0 i}=\chi_{i+1}, k=i$ and go back to Step 3, else go to Step exit.

Step 5. Let

$$
\begin{aligned}
\dot{\chi}_{i} & =\chi_{i+1}+\eta_{1 i}(\tilde{y})+\eta_{2 i}(\tilde{y}) u, \quad i \in[1, \ell-1] \\
\dot{\chi}_{\ell} & =\eta_{0, \ell}(\chi)+\eta_{1 \ell}(\tilde{y})+\eta_{2 \ell}(\tilde{y}) u \\
\dot{x} & =f(x)+g(x) u
\end{aligned}
$$

\footnotetext{
${ }^{2}$ The assumption that $\eta_{1}, \eta_{2}$ and $\varphi$ in (2) are smooth, guarantees that if a solution exists then $\beta_{y}(y), \mathcal{P}_{1}(y)$ and $\mathcal{P}_{2}(y)$ in the algorithm are integrable.
} 
be the extended system computed above. Consider $r_{11}^{e}=e^{-\chi_{1}} \tilde{r}_{1}^{T} \frac{\partial}{\partial x}$ which is the solution to (9) with respect to the set of outputs $y_{1}^{e}=e^{\chi_{1}} \tilde{y}, y_{1+i}=\chi_{i}, i \in[1, \ell]$. By construction $\left(r_{11}^{e}, \cdots, r_{1 n}^{e}\right)=\left(\begin{array}{c}R \\ 0\end{array}\right)$. Compute the $n$ independent functions $\lambda(x, \chi)=\left(\lambda_{1}(x, \chi), \cdots, \lambda_{n}(x, \chi)\right)^{T}$, where $\frac{\partial \lambda(x, \chi)}{\partial x}=R^{-1}$. In the coordinates $\left(\tilde{\chi}^{T}, z^{T}\right)^{T}=$ $\left(\chi^{T}, \lambda(x, \chi)^{T}\right)^{T}$ the extended system is in the canonical observer form up to input and output injection.

Step exit The system does not admit the canonical observer form up to input output injection under regular compensator and regular output transformation.

The main result can now be stated in terms of a necessary and sufficient condition under which the problem can be solved.

Theorem 3: Given the observable system (1), (2), there exists a dynamic compensator of dimension $\ell \geq 0$ of the form (3) and a regular output transformation of the form (4) such that the extended system (1), (3) is equivalent to the canonical observer form up to input and output injection if and only if the algorithm ends at Step 5. Furthermore the computed compensator is of minimal dimension.

Proof: If the algorithm ends at Step 5, then a compensator has been computed together with the appropriate change of coordinates which transforms the extended system in the canonical observer form up to input and output injection, thus proving the sufficiency part. As for the necessity the algorithm is based on the necessary conditions enounced in Theorem 2 with the compensator already written in the form (27). At Step 3, the compensator is updated by adding a dynamics of the form (29), where $\eta_{1 i}(\tilde{y})$ and $\eta_{2 i}(\tilde{y})$ are computed in order to satisfy the conditions on the coefficients $\tilde{\Gamma}_{j-i}^{j}$ and $\tilde{\Theta}_{i-1}$ respectively given in Theorem 2 , as a consequence the algorithm cannot end at step exit if a compensator exists. Step 4 guarantees that the computed compensator is of minimal order by checking each time the compensator is updated, if there exists an $\eta_{0 i}(\chi)$ which guarantees that Theorem 2 is satisfied.

Example 1: Consider the following system

$$
\begin{aligned}
& \dot{x}_{1}=x_{2}, \dot{x}_{2}=x_{3}, \dot{x}_{3}=x_{4}+2 x_{2} u \\
& \dot{x}_{4}=x_{1}^{2} x_{4}+x_{2} x_{3}-\frac{2}{5} x_{2}^{3}+x_{3} u, \quad y=x_{1}
\end{aligned}
$$

Step 0. Standard computations lead to $r_{1}=\frac{\partial}{\partial x_{4}}, r_{2}=\frac{\partial}{\partial x_{3}}+x_{1}^{2} \frac{\partial}{\partial x_{4}}, r_{3}=\frac{\partial}{\partial x_{2}}+x_{1}^{2} \frac{\partial}{\partial x_{3}}+\left(x_{1}^{4}+x_{2}-2 x_{1} x_{2}\right) \frac{\partial}{\partial x_{4}}$ and $r_{4}=\frac{\partial}{\partial x_{1}}+x_{1}^{2} \frac{\partial}{\partial x_{2}}+\left(x_{1}^{4}+x_{2}-4 x_{1} x_{2}\right) \frac{\partial}{\partial x_{3}}+\left(x_{1}^{6}+2 x_{1}^{2} x_{2}-6 x_{1}^{3} x_{2}+\frac{4}{5} x_{2}^{2}+2 x_{1} x_{3}\right) \frac{\partial}{\partial x_{4}}$.

Since $\left[r_{1}, r_{2}\right]=\left[r_{1}, r_{3}\right]=0$, we go to next step

Step 1. $\left[r_{1}, r_{4}\right]=\left[r_{2}, r_{4}\right]=0, \mathcal{L}_{0}^{1}(0,0)=-2$ and $\Gamma_{1}^{1}=0$, so that $\beta(y)=0$ which implies that $\tilde{y}=y=x_{1}$, and we go to next step.

Step 2. $\left[r_{3}, r_{4}\right]=\left(1-6 x_{1}\right) r_{2}+\frac{18}{5} x_{2} r_{1}$. Consequently $\Gamma_{1}^{1}=\Gamma_{2}^{2}=\Gamma_{3}^{3}=0$ and we set $\alpha\left(\tilde{y}, \chi_{1}\right)=e^{-\chi_{1}}$, and $k=0$. Moreover $\Gamma_{2}^{3}=1-6 x_{1}$ while $\Gamma_{1}^{3}=\frac{18}{5} x_{2}$, whereas $\Gamma_{1}^{2}=0$.

Step 3. We set $i=1$ and $\dot{\chi}_{1}=\eta_{01}(\chi)+\eta_{11}(\tilde{y})+\eta_{21}(\tilde{y}) u$.

Step 3a. $\mathcal{L}_{1}^{3}(0,0)=-5$. Accordingly

$$
\mathcal{P}_{1}=-\Gamma_{2}^{3} / \mathcal{L}_{1}^{3}(0,0)=\left(1-6 x_{1}\right) / 5=(1-6 \tilde{y}) / 5
$$

so that $\eta_{11}=\int \mathcal{P}_{1} d \tilde{y}=\left(x_{1}-3 x_{1}^{2}\right) / 5$

Step 3b. Since $g=\left(0,0,2 x_{2}, x_{3}\right)^{T},\left[r_{1}, g\right]=0$, so that $\Theta_{0}=0$ and consequently $\eta_{21}=0$.

Step 4. There doesn't exist a $\eta_{01}\left(\chi_{1}\right)$ which ensures that $\Gamma_{1}^{3}=Q_{3}^{2}\left(F^{e}, \chi_{1}\right)$. In fact it should satisfy the equation $\frac{\partial \eta_{01}\left(\chi_{1}\right)}{\partial \chi_{1}}=-x_{1}^{2}$. We thus go back to Step 3 with $k=1$ and $\eta_{01}=\chi_{2}$.

Step 3. We set $i=2$ and $\dot{\chi}_{2}=\eta_{02}(\chi)+\eta_{12}(\tilde{y})+\eta_{22}(\tilde{y}) u$.

Step 3a. $\mathcal{L}_{2}^{3}(0,0)=-2$. Accordingly

$$
\mathcal{P}_{2}=\left(\Gamma_{1}^{3}-\sum_{l=0}^{1} L_{f}^{1-l}\left(\frac{\partial \eta_{11}(\tilde{y})}{\partial \tilde{y}}\right) \mathcal{L}_{2}^{3}(1, l)\right) / \mathcal{L}_{2}^{3}(0,0)=-\frac{1-6 x_{1}}{5} x_{1}^{2}
$$


so that $\eta_{12}(\tilde{y})=\int \mathcal{P}_{2} d \tilde{y}=\frac{3}{10} x_{1}^{4}-\frac{1}{15} x_{1}^{3}$.

Step 3b. Since $\left[r_{2}, g\right]=r_{1}$, we get that $\Theta_{1}=1$. Consequently we have that $\eta_{22}=\Theta_{1}-L_{g} \eta_{11}=1$

Step 4. $\left[r_{3}, g\right]=2 r_{2}-x_{1}^{2} r_{1}=2 \Theta_{1} r_{2}+\Theta_{2} r_{1}$. Consequently $\Theta_{2}=-x_{1}^{2}$. We have to check if there exists $\eta_{02}\left(\chi_{1}, \chi_{2}\right)$ such that $L_{G e} \eta_{02}=-\Theta_{2}-L_{g} L_{f} \eta_{11}-L_{g} \eta_{12}$. Such a function doesn't exists since it should satisfy the relation $\frac{\partial \eta_{02}}{\partial \chi_{1}} \eta_{21}+\frac{\partial \eta_{02}}{\partial \chi_{2}} \eta_{22}=\frac{\partial \eta_{02}}{\partial \chi_{2}}=x_{1}^{2}$. We thus go back to Step 3 with $k=2$ and $\eta_{02}=\chi_{3}$.

Step 3. We set $i=3$ and $\dot{\chi}_{3}=\eta_{03}(\chi)+\eta_{13}(\tilde{y})+\eta_{23}(\tilde{y}) u$.

Since $i+1=4>n-1=3$ all the conditions concerning the autonomous part are satisfied and $\eta_{13}(\tilde{y})=0$. From $\Theta_{2}=-x_{1}^{2}$ we get that $\eta_{23}(\tilde{y})=-\Theta_{2}-L_{g} \eta_{12}-L_{g} L_{f} \eta_{11}=x_{1}^{2}$. Since the compensator has dimension $n-1$ we can set $\eta_{03}=0 .^{3}$

Step 5. The computed compensator is then

$$
\dot{\chi}_{1}=\chi_{2}+\left(x_{1}-3 x_{1}^{2}\right) / 5, \quad \dot{\chi}_{2}=\frac{3}{10} x_{1}^{4}-\frac{1}{15} x_{1}^{3}+u, \quad \dot{\chi}_{3}=x_{1}^{2} u
$$

Accordingly the associated change of coordinates is

$$
\begin{aligned}
& z_{1}=e^{\chi_{1}}\left(x_{4}-\chi_{2} x_{3}-\frac{1}{5} x_{1} x_{3}-\frac{2}{5} x_{1}^{2} x_{3}+\chi_{3} x_{2}+\chi_{2}^{2} x_{2}-3 \chi_{2} \chi_{3} x_{1}\right) \\
& +e^{\chi_{1}}\left(\frac{1}{25} x_{1}^{2} x_{2}-\frac{3}{10} \chi_{3} x_{1}^{2}+\frac{4}{15} \chi_{3} x_{1}^{3}+\frac{2}{5} \chi_{2} x_{1} x_{2}-\frac{1}{5} \chi_{2} x_{1}^{2} x_{2}-\frac{8}{75} x_{1}^{3} x_{2}\right) \\
& +e^{\chi_{1}}\left(\frac{3}{50} x_{1}^{4} x_{2}-\frac{2}{5} x_{2}^{2}+\frac{2}{5} x_{1} x_{2}^{2}+\frac{13}{100} \chi_{2} x_{1}^{4}+\frac{9}{625} x_{1}^{5}-\frac{39}{250} \chi_{2} x_{1}^{5}-\chi_{2}^{3} x_{1}\right) \\
& +e^{\chi_{1}}\left(\frac{4}{15} \chi_{2}^{2} x_{1}^{3}-\frac{157}{4500} x_{1}^{6}+\frac{12}{875} x_{1}^{7}-\frac{3}{10} \chi_{2}^{2} x_{1}^{2}-\frac{1}{25} \chi_{2} x_{1}^{3}-\frac{1}{500} x_{1}^{4}\right) \\
& z_{2}=e^{\chi_{1}}\left(x_{3}+3 \chi_{3} x_{1}-2 \chi_{2} x_{2}-\frac{2}{5} x_{1} x_{2}+\frac{1}{5} x_{1}^{2} x_{2}+3 \chi_{2}^{2} x_{1}\right) \\
& \quad+e^{\chi_{1}}\left(\frac{1}{25} x_{1}^{3}-\frac{13}{100} x_{1}^{4}+\frac{3}{5} \chi_{2} x_{1}^{2}-\frac{8}{15} x_{1}^{3} \chi_{2}+\frac{39}{250} x_{1}^{5}\right) \\
& z_{3}=e^{\chi_{1}}\left(x_{2}-3 \chi_{2} x_{1}-\frac{3}{10} x_{1}^{2}+\frac{4}{15} x_{1}^{3}\right), \\
& z_{4}=x_{1} e^{\chi_{1}}, \tilde{\chi}_{i}=\chi_{i}, i \in[1,3] .
\end{aligned}
$$

In these coordinates the system is in the form (5). The algorithms in [28], [34], [8] fail in determining a solution.

\section{CONCLUSIONS}

In the present paper we have given necessary and sufficient conditions for the existence of a dynamic compensator such that the extended system is equivalent under change of coordinates and regular output transformations to a linear observable system up to input output injection. The given conditions are constructive and allow to characterize completely the structure of the controller, thus leading to the computation of a solution. An algorithm has also been proposed for the computation of a solution of minimal dimension if it exists. Further investigations will concern non regular solutions.

\section{REFERENCES}

[1] V. Andrieu and L. Praly, On the existence of a Kazantzis-Kravaris/Luenberger Observer, SIAM J. Con. Opt. 45, 432-456, 2006.

[2] J. Back and J.H. Seo, An Algorithm for system immersion into nonlinear observer form: SISO case, Automatica, 42, 321-328, 2006.

[3] J. Back, K.T. Yu and J.H. Seo, Dynamic Observer Error Linearization, Automatica, 42, 2195 - 2200, 2006.

[4] G. Besançon, H.Hammouri, S. Benamor State equivalence of discrete-time nonlinear control systems to state affine form up to input/output injection, SCL, 33, 1-10, 1998.

[5] G. Besançon, On output transformations for state linearization up to output injection, IEEE TAC, 44, 1975 - 1981, 1999.

[6] D. Bestle and M. Zeitz, Canonical form observer design for non-linear time-variable systems, IJC, 419-431, 1983.

[7] D. Boutat, Geometrical conditions for observer error linearization via $0 \rightarrow 1 \rightarrow \cdots \rightarrow(N-2)-\int$, Proc. of 7th IFAC Nolcos 2007, pp. 1020-1025, Pretoria, South Africa, 2007.

[8] D. Boutat and K. Busawon, On the transformation of nonlinear dynamical systems into the extended nonlinear observable canonical form, IJC, 84, 94-106, 2011.

\footnotetext{
${ }^{3} \eta_{03}$ may be chosen to achieve stabilization of the compensator for $y=u=0$.
} 
[9] C. Califano, S. Monaco and D. Normand-Cyrot, Canonical observer forms for multi-output systems up to coordinate and output transformations in discrete time, Automatica, 45, 2483-2490, 2009.

[10] C. Califano, S. Monaco and D. Normand-Cyrot, On the observer design through output scaling in discrete-time, Proc. of ACC 2010, Art. N.5530727, 5290-5295; Baltimore, MD, USA, 2010

[11] C. Califano, L.A. Marquez-Martinez and C.H. Moog, On the observer canonical form for time-delay systems, IFAC-PapersOnline, 18, PART 1, 2011, 3855-3860, 18th IFAC World Congress; Milan; Italy 2011

[12] A. Glumineau, C. H. Moog, and F. Plestan, New algebro-geometric conditions for the linearization by input-output injection, IEEE TAC, 41, $598-603,1996$.

[13] H. Hammouri and M. Kinnaert, A new procedure for time-varying linearization up to output injection, SCL, 28, 151-157, 1996.

[14] M. Hou and A.C. Pugh, Observer with linear error dynamics for nonlinear multi-output systems, SCL, 37, 1-9, 1999.

[15] H.J.C. Huijberts, On existence of extended observers for nonlinear discrete-time systems, Lect. Notes in Cont. and Info. Sci., 244, 73-92, Springer-Verlag, 1999

[16] A. Isidori, Nonlinear Control Systems, Springer Verlag, 3rd Ed., 1995

[17] P. Jouan, Immersion of nonlinear systems into linear systems modulo output injection, Siam J. Cont. Opt., 41, 1756-1778, 2003.

[18] V. Kaparin U. Kotta, T. Mullari, Extended observer form: simple existence conditions, Int J Control, 86, pp. 794-803 ,2013

[19] I. Karafyllis and C. Kravaris, On the observer problem for discrete-time control systems, IEEE TAC, 52, 12-25, 2007.

[20] N. Kazantzis and C. Kravaris, Nonlinear Observer Design using Lyapunov's auxiliary theorem, SCL, 34, 241-247, 1998.

[21] H. Keller, Non-linear observer design by transformation into a generalized observer canonical form , IJC, 46, 1915-1930, 1987.

[22] A.J. Krener and A. Isidori, Linearization by output injection and nonlinear Observers, SCL, 3, 47-52, 1983.

[23] A.J. Krener and W. Respondek, Nonlinear Observers with linearizable error dynamics, SIAM J. Cont. Opt., 23, 197-216, 1985.

[24] A.J. Krener and M. Xiao, Nonlinear observer design in the Siegel domain, SIAM J. Cont. Opt., 41, 932-953, 2002.

[25] W. Lin and C.I. Byrnes, Remarks on Linearization of discrete-time autonomous systems and nonlinear observer design, SCL, 25, 31-40, 1995.

[26] L.A. Marquez-Martinez, C.H. Moog and M. Velasco-Villa, Observability and observers for nonlinear systems with time delay, Kybernetika, 38, 445-456, 2002.

[27] S. Monaco and D. Normand-Cyrot, Linearization by Output Injection under Approximate Sampling, EJC, 15, 205-217, 2009.

[28] D. Noh, N.H. Jo, J.H. Seo, Nonlinear Observer Design by Dynamic observer Error Linearization, IEEE TAC., 49, 1746-1750, 2004.

[29] F. Plestan, and A. Glumineau, Linearization by generalized input-output injection, SCL, 31, 115-128, 1997.

[30] W. Respondek, A. Pogromsky and H. Nijmeijer, Time scaling for observer design with linearizable error dynamics, Autom., 277-285, 2004.

[31] I. Souleiman, A. Glumineau, and G. Schreirer. Direct transformation of nonlinear systems into state affine MISO form and nonlinear observers design, IEEE TAC, 48, 2191-2196, 2003.

[32] Y. Wang and A. Lynch, A block triangular form for nonlinear observer design, IEEE TAC, 1803-1808, 2006.

[33] X-H Xia and W-B. Gao, Nonlinear observer design by observer error linearization, SIAM J. Cont. Opt., 27, 199-216, 1989.

[34] J. Yang, J. Back, J.H. Seo, H. Shim, Reduced-order Dynamic observer error linearization, Proc. 8th IFAC Nolcos 2010, 915-920, Italy, 2010.

[35] K.T. Yu, J. Back and J.H. Seo, Constructive algorithm for dynamic Observer Error Linearization via integrators: single output case, International Journal of Robust and nonlinear Control, 17, 25 - 49, 2007.

[36] J. Zhang G. Feng, H. Xu, Observer design for nonlinear discrete-time systems: immersion and dynamic observer error linearization techniques, Int J Robust and Nonlinear Control, 20, 504-514, 2010.

[37] G. Zheng, J.P. Barbot, D. Boutat, T. Floquet, J.P. Richard, On observation of time-delay systems with unknown inputs, IEEE TAC, 56, 1973-1978, 2011.

\section{APPENDIX}

Proof of Proposition 4. Assume that the problem admits a solution. By Proposition 3 let the dynamic compensator (3) with extended output $\tilde{y}^{e}=\left(\varphi(y, \xi), \xi_{1}, \cdots, \xi_{\ell}\right)^{T}$, be a possible solution where $\frac{\partial \varphi(y, \xi)}{\partial y}=e^{\beta_{I, y}(y)+\beta_{0}(\xi)} c_{0}$. If $\beta_{0}(\xi)=0$ then the system admits the canonical observer form up to input-output injection with respect to the transformed output $\tilde{y}_{1}=\int e^{\beta_{I, y}(y)} c_{0} d y+c o s t$, so that $\bar{\ell}=0$. In fact, by Proposition 2 and eqs. (10), (12), $\alpha(y, \xi)=\alpha(y)=e^{-\beta_{I, y}(y)} / c_{0}$ and denoting by $\tilde{R}_{1 j}(x)=\sum_{i=0}^{j-1} \mathcal{C}_{i}^{j-1}(-1)^{j-1-i} L_{f}^{j-i-1} \alpha(x) r_{i+1}(x)$ the vector fields associated to $\tilde{y}, \tilde{r}_{1 j}^{e}(x, \xi)=\tilde{R}_{1 j}^{T}(x) \frac{\partial}{\partial x}$. By assumption $\left[\tilde{r}_{1 j}^{e}(x), \tilde{r}_{1 i}^{e}(x)\right]=\left[\tilde{R}_{1 j}^{T}(x) \frac{\partial}{\partial x}, \tilde{R}_{1 i}^{T}(x) \frac{\partial}{\partial x}\right]=0$, $\forall i, j \in[1, n]$. Moreover, $\forall j \in[1, n-1],\left[g^{e}(x, \xi), \tilde{r}_{1 j}^{e}(x)\right]=\left[\eta_{2}^{T}(\xi, y) \frac{\partial}{\partial \xi}+g^{T}(x) \frac{\partial}{\partial x}, \tilde{R}_{1 j}^{T}(x) \frac{\partial}{\partial x}\right]=0$, which implies that $\forall j \in[1, n-1],\left[g(x), \tilde{R}_{1 j}(x)\right]=0$, thus proving that the problem is solvable by output tranformation only.

Assume now that $\beta_{0}(\xi) \neq 0$ and assume without loss of generality $\beta_{\xi_{1}}=\frac{\partial \beta_{0}}{\partial \xi_{1}} \neq 0$ (this can be achieved after a possible renaming of the variables). Then setting $\chi_{1}=\beta_{0}(\xi)$, one gets that in the coordinates $\left(\chi_{1}, \xi_{2}, \cdots, \xi_{\ell}\right)$ the dynamic compensator reads

$$
\begin{aligned}
& \dot{\chi}_{1}=\bar{\eta}_{11}\left(\chi_{1}, \xi_{2} \cdots, \xi_{\ell}, y\right)+\bar{\eta}_{21}\left(\chi_{1}, \xi_{2} \cdots, \xi_{\ell}, y\right) u \\
& \dot{\xi}_{i}=\bar{\eta}_{1, i}\left(\chi_{1}, \xi_{2} \cdots, \xi_{\ell}, y\right)+\bar{\eta}_{2 i}\left(\chi_{1}, \xi_{2} \cdots, \xi_{\ell}, y\right) u, i \in[2, \ell] .
\end{aligned}
$$

with output functions $\tilde{y}_{1}^{e}=\varphi\left(y, \chi_{1}, \xi_{2}, \cdots \xi_{\ell}\right), \hat{y}_{2}^{e}=\beta_{0}^{-1}\left(\chi_{1}, \xi_{2}, \cdots, \xi_{\ell}\right), \hat{y}_{1+i}^{e}=\xi_{i}$ for $i \in[2, \ell]$. Due to Proposition 1 , the system admits the canonical form (5) also with respect to the output functions $\tilde{y}_{1}=\varphi\left(y, \chi_{1}, \xi_{2}, \cdots \xi_{\ell}\right)$, $\tilde{y}_{2}=\chi_{1}, \hat{y}_{1+i}=\xi_{i}$ for $i \in[2, \ell]$. Take then $H_{1}=\left(h(x), \chi_{1}, \xi_{2}, \cdots, \xi_{\ell}\right)^{T}$ as output functions and note that since in the new coordinates the output transformation satisfies $\frac{\partial \varphi\left(y, \chi_{1}, \xi\right)}{\partial y}=e^{\beta_{I, y}(y)} e^{\chi_{1}} c_{0}$ then the associated vector 
$\beta\left(y, \chi_{1}, \xi_{2}, \cdots, \xi_{\ell}\right)=\left[\beta_{y}, 1,0 \cdots 0\right]$. As a consequence for $j>0, \beta^{(j)}=\left[\beta_{y}^{(j)}, 0,0 \cdots 0\right]$. Since Theorem 2 is satisfied, in (15), $\Gamma_{j-i}^{j}$ which depends on the variable $x$ only, reads

$$
\Gamma_{j-i}^{j}=(-1)^{i}\left(\sum_{k=0}^{i} \mathcal{C}_{j-1-i}^{j-1-k} \beta_{y}^{(i-k)} Q_{j}^{k}(f, h)+Q_{j}^{i}\left(F^{e}, \chi_{1}\right)\right)
$$

with $Q_{j}^{k}(f, h)$ and $Q_{j}^{i}\left(F^{e}, \chi_{1}\right)$ defined by (17). For $i=1, d\left(L_{F e}^{n-j+1} \chi_{1}\right) r_{j}^{T} \frac{\partial}{\partial x}=d\left(L_{F^{e}} \chi_{1}\right) r_{n}^{T} \frac{\partial}{\partial x}=\frac{\partial \bar{\eta}_{11}\left(y, \chi_{1}, \xi_{2}, \cdots, \xi_{\ell}\right)}{\partial y}$, since $\beta_{y}$ is known, we can obtain $\beta_{y}^{(1)}$ and considering $\mathcal{L}_{i}^{j}(p, l)$ defined by (28), we get from (31)

$$
-\frac{\partial \tilde{\eta}_{11}(\cdot)}{\partial y} \mathcal{L}_{1}^{j}(0,0)=\Gamma_{j-1}^{j}(x)+\sum_{k=0}^{1} \mathcal{C}_{j-2}^{j-1-k} \beta_{y}^{(1-k)} Q_{j}^{k}(f, h)
$$

Since the right-hand side depends on the $x$ variable only, choosing a $j>1$ such that $\mathcal{L}_{1}^{j}(0,0) \neq 0$, necessarily $\frac{\partial \bar{\eta}_{11}\left(y, \chi_{1}, \xi_{2}, \cdots, \xi_{\ell}\right)}{\partial y}=\psi_{1}(y)$. Consequenly $\bar{\eta}_{11}\left(\chi_{1}, \xi_{2}, \cdots, \xi_{\ell}, y\right)=\tilde{\eta}_{01}\left(\chi_{1}, \xi_{2}, \cdots, \xi_{\ell}\right)+\tilde{\eta}_{11}(y)$ with $\tilde{\eta}_{11}(y)=$ $\int \psi_{1}(y) d y$.

Analogously $\Theta_{0}$ in (19) reads

$$
\Theta_{0}(x)=L_{\bar{g}^{e}} \log \left(\alpha\left(y, \chi_{1}\right)\right)=-L_{\bar{g}^{e}}\left(\beta_{I, y}+\chi_{1}\right)=-L_{\bar{g} e}\left(\beta_{I, y}\right)-\bar{\eta}_{21}(\cdot)
$$

so that $\bar{\eta}_{21}(\cdot)=-L_{\bar{g}^{e}}\left(\beta_{I, y}\right)-\Theta_{0}(x)$. Since the right handside depends on $x$ only necessarily $\bar{\eta}_{21}(\cdot)=\tilde{\eta}_{21}(y)$. If $\tilde{\eta}_{01}(\cdot)$ depends on $\xi_{j}, j>1$, we can set $\tilde{\eta}_{01}=\chi_{2}$, and, assuming without loss of generality that $\frac{\partial \tilde{\eta}_{01}}{\partial \xi_{2}} \neq 0$, consider the coordinates $\left(\chi_{1}, \chi_{2}, \xi_{3}, \cdots, \xi_{\ell}\right)$ in which the dynamic compensator reads

$$
\begin{aligned}
\dot{\chi}_{1} & =\chi_{2}+\tilde{\eta}_{11}(y)+\tilde{\eta}_{21}(y) u \\
\dot{\chi}_{2} & =\hat{\eta}_{12}\left(\chi_{1}, \chi_{2}, \xi_{3} \cdots, \xi_{\ell}, y\right)+\hat{\eta}_{22}\left(\chi_{1}, \chi_{2}, \xi_{3} \cdots, \xi_{\ell}, y\right) u \\
\dot{\xi}_{j} & =\hat{\eta}_{1 j}\left(\chi_{1}, \chi_{2}, \xi_{3} \cdots, \xi_{\ell}, y\right)+\hat{\eta}_{2 j}\left(\chi_{1}, \chi_{2}, \xi_{3} \cdots, \xi_{\ell}, y\right) u, j \in[3, \ell]
\end{aligned}
$$

and we can take as output $H_{2}=\left(h(x), \chi_{1}, \chi_{2}, \xi_{3} \cdots, \xi_{\ell}\right)^{T}$.

Consider now $\Gamma_{j-2}^{j}$ the coefficient of $r_{j-2}$ in equation (15). From (31) denoting by $\hat{F}^{e}$ the drift in the new coordinates, one gets

$$
\Gamma_{j-2}^{j}(x)=\sum_{k=0}^{2} \mathcal{C}_{j-3}^{j-1-k} \beta_{y}^{(2-k)} Q_{j}^{k}(f, h)+Q_{j}^{2}\left(\hat{F}^{e}, \chi_{1}\right)
$$

Developing the previous expression, we have that

$$
\begin{aligned}
& \mathcal{L}_{2}^{j}(0,0) \frac{\partial \hat{\eta}_{12}(y, \chi, \xi)}{\partial y}=\Gamma_{j-2}^{j}-\sum_{k=0}^{2} \mathcal{C}_{j-3}^{j-1-k} \beta_{y}^{(2-k)} Q_{j}^{k}(f, h) \\
& -\left[(-1)^{n-j} \mathcal{C}_{n-j+2}^{n-1} d\left(L_{f}^{n-j+1} \tilde{\eta}_{11}\right) r_{j}^{T} \frac{\partial}{\partial x}-\mathcal{C}_{2}^{j-1} d\left(L_{f} \tilde{\eta}_{11}\right) r_{n}^{T} \frac{\partial}{\partial x}\right]
\end{aligned}
$$

Choosing a value of $j>2$ such that $\mathcal{L}_{2}^{j}(0,0) \neq 0$, allows the computation of $\hat{\eta}_{12}(\cdot, y)$, and since the righthand side depends on $x$ only then $\frac{\partial \hat{\eta}_{12}(\cdot, y)}{\partial y}=\psi_{2}(y)$ so that denoting by $\tilde{\eta}_{12}(y)=\int \psi_{2}(y) d y$ then $\hat{\eta}_{12}(\cdot, y)=$ $\tilde{\eta}_{02}\left(\chi_{1}, \chi_{2}, \xi_{3}, \cdots, \xi_{\ell}\right)+\tilde{\eta}_{12}(y)$.

Let $\hat{g}^{e}(x, \chi, \xi)$ be $g^{e}(\cdot)$ in the new coordinates. From (19),

$$
\begin{aligned}
\Theta_{1} & =L_{\hat{g}^{e}} L_{\hat{F}^{e}}\left(\beta_{I, y}+\chi_{1}\right)=L_{\hat{g}^{e}} L_{\hat{F}^{e}}\left(\beta_{I, y}\right)+L_{\hat{g}^{e}}\left(\chi_{2}+\tilde{\eta}_{11}(y)\right) \\
& =L_{\hat{g}^{e}} L_{\hat{F}^{e}}\left(\beta_{I, y}\right)+L_{\hat{g}^{e}}\left(\tilde{\eta}_{11}(y)\right)+\hat{\eta}_{22}(\cdot)
\end{aligned}
$$

Thus, $\tilde{\eta}_{22}(\cdot)=\Theta_{1}-L_{\hat{g}^{e}} L_{\hat{F}^{e}}\left(\beta_{I, y}\right)-L_{\hat{g}^{e}}\left(\tilde{\eta}_{11}(y)\right)=\tilde{\eta}_{22}(y)$. Iterating the reasoning one gets that there exists an index $\bar{\ell} \leq \ell$ such that the dynamic compensator can be written as

$$
\begin{aligned}
& \dot{\chi}_{i}=\chi_{i+1}+\tilde{\eta}_{1 i}(y)+\tilde{\eta}_{2 i}(y) u, \quad i \in[1, \bar{\ell}-1] \\
& \dot{\chi}_{\bar{\ell}}=\tilde{\eta}_{0, \bar{\ell}}(\chi)+\tilde{\eta}_{1 \bar{\ell}}(y)+\tilde{\eta}_{2 \bar{\ell}}(y) u \\
& \dot{\xi}_{j}=\tilde{\eta}_{1 j}\left(\chi, \xi_{\bar{\ell}+1}, \cdots, \xi_{\ell}\right)+\tilde{\eta}_{2 j}\left(\chi, \xi_{\bar{\ell}+1}, \cdots, \xi_{\ell}\right) u, j \in[\bar{\ell}+1, \ell]
\end{aligned}
$$

where for $i \in[1, \bar{\ell}]$, the $\tilde{\eta}_{1 i}(y)$ 's can be computed by integrating the function $\psi_{i}(y)$ defined from the coefficient $\Gamma_{j-i}^{j}$ given by (16), while the $\tilde{\eta}_{2 i}(y)$ 's are defined by the coefficient $\Theta_{i}$ given by (19). 
We show now that if $\bar{\ell}<\ell$, the dynamics (33) can be neglected, thus reducing the dimension of the dynamic compensator from $\ell$ to $\bar{\ell}$. Consider the extended system (32)-(7), and consider $\tilde{r}_{11}^{e}=\alpha\left(y, \chi_{1}\right) r_{1}^{T} \frac{\partial}{\partial x}$, with $\alpha\left(y, \chi_{1}\right)$ and $r_{1}$ computed above. Accordingly, compute $\tilde{r}_{1 j}^{e}$ for $j \in[1, n]$. By construction $\left[\tilde{r}_{1 i}^{e}, \tilde{r}_{1 j}^{e}\right]=0$ for $i, j \in[1, n]$. Since the distribution $\Delta^{e}=\operatorname{span}\left\{\tilde{r}_{11}^{e}, \cdots, \tilde{r}_{1 n}^{e}\right\}$ is nilpotent, define the change of coordinates $\left(\tilde{\chi}^{T}, z^{T}\right)^{T}=\left(\chi^{T}, \lambda^{T}(x, \chi)\right)^{T}$ such that $d \chi \Delta^{e}=0$ while $d \lambda(x, \chi) \Delta^{e}=I$. In these new coordinates $F^{e}(\tilde{\chi}, z)=\left(\tilde{\eta}_{0}^{T}(\tilde{\chi})+\tilde{\eta}_{1}^{T}(y), \quad \tilde{f}^{T}(\tilde{\chi}, z)\right)^{T}$ and $G^{e}(\tilde{\chi}, z)=\left(\tilde{\eta}_{2}^{T}(y), \quad \tilde{g}^{T}(\tilde{\chi}, z)\right)^{T}$. Then, for $i \in[1, n-1], \frac{\partial F^{e}(\tilde{\chi}, z)}{\partial z_{i}}=\left[\tilde{r}_{1 i}^{e}, F^{e}(\tilde{\chi}, z)\right]=\tilde{r}_{1, i+1}^{e}=\frac{\partial}{\partial z_{i+1}}$, $\frac{\partial G^{e}(\tilde{\chi}, z)}{\partial z_{i}}=\left[\tilde{r}_{1 i}^{e}, G^{e}(\tilde{\chi}, z)\right]=0$ and $z_{n}=\hat{y}_{1}^{e}=e^{\chi_{1}} \tilde{y}+\varphi_{0}\left(\chi_{2}, \cdots, \chi_{\bar{\ell}}\right)$, which proves that in the new coordinates the system reads

$$
\begin{aligned}
\dot{\tilde{\chi}} & =\tilde{\eta}_{0}(\tilde{\chi})+\tilde{\eta}_{1}(y)+\tilde{\eta}_{2}(y) u \\
\dot{z} & =A z+\psi_{1}(y, \chi)+\psi_{2}(y, \chi) u, \quad \hat{y}=\varphi(y, \chi)=z_{n}
\end{aligned}
$$

Finally since the dynamics of the compensator is defined by the $\Gamma_{j-i}^{j}$ 's $j \in[1, n-1]$ and the $\Theta_{l}$ 's $l \in[0, n-1]$, then its maximum necessary dimension is $n-1$. 